\title{
Probabilistic Study of the Impact on the Network Equipment of Changing Load Profiles in Modern Low Voltage Grids
}

\author{
J-F. Toubeau ${ }^{1}$, V. Klonari ${ }^{1}$, Z. De Grève ${ }^{1}$, J. Lobry $^{1}$ and F. Vallée ${ }^{2}$ \\ ${ }^{1}$ Department of Electrical Engineering \\ ${ }^{2}$ Department of General Physics \\ Faculté Polytechnique, University of Mons \\ Boulevard Dolez 31, B-7000 Mons (Belgium) \\ Phone/Fax number: +0032 65 374123, e-mail: Jean-Francois.TOUBEAU@umons.ac.be
}

\begin{abstract}
The paper proposes a measurement-based method for the long-term analysis of the low-voltage (LV) distribution network. This work focuses on line power flows susceptible to cause a premature deterioration of cables and on power exchanged at the MV/LV transformer that can potentially induce adverse repercussions on its lifetime. Such estimations are indeed essential as the distribution systems are more and more frequently subject to stress conditions due to the increase of photovoltaic (PV) installations and significant loads such as electric vehicles. In this context, using a probabilistic approach is more beneficial than worst-case scenarios that yield much more restrictive conclusions. The approach is implemented in a pseudo-sequential Monte-Carlo environment and is based on quarter-hourly energy flows recorded by smart meters installed at some LV customers of the Belgian distribution network. These devices are currently located in some scarce selected areas but the penetration rate is expected to massively grow in the future. With the increase of collected measurements, this analysis tool will therefore allow Distribution System Operators (DSOs) to accurately assess important parameters for a secure and efficient operation of the network.
\end{abstract}

\section{Key words}

Dispersed generation, electric vehicles, probabilistic study.

\section{Introduction}

The recent worldwide awareness of the need to substantially decrease the carbon emission involves a renewed vision of the energy sector. This is reflected particularly by a massive introduction of power generation based on renewable energy sources but also by the development of electrification in sectors as heating and transportation. This involves an important change in low-voltage (LV) networks with, not only a massive roll-out of distributed generation, but also with the emergence of significant loads such as heat pumps and electric vehicles (EVs). Such a situation leads to several adverse impacts for the distribution system operator (DSO) and, as a result, for the LV customers.

The major issue is the important risk of voltage violation that can accelerate the aging of the electrical equipment of the different consumers. As the dispersed generation depends on weather conditions and the load profile substantively fluctuates over time, the network is subject to both undervoltage and overvoltage issues depending on the period of the time envisaged. Modern LV grids are also subject to an increased risk of voltage imbalance between phases as residential customers are currently randomly connected to the network phases without a global balancing strategy from the DSO. This situation had no significant impact in the past due to a natural repartition of the different clients but could lead to unacceptable outcomes with the single-phase connection of photovoltaic (PV) installations and the important loading increase at some places.

This change of paradigm in distribution grids impacts also naturally the power flows throughout the different feeders. The network operates indeed closer to its limits which may induce unintended line congestions as well as an extensive increase of the transformer load. The problem of excessive current flows would require upgrading the feeders but such a solution is not economically viable for DSOs, especially with the current trend to install underground power lines. The loading rise at the MV/LV substation has also a detrimental effect on the transformer as it can potentially reduce its lifetime [1]. It is thus important for the DSO to quantify this aging in order to be able to make the necessary arrangements.

Generally, the accurate assessment of these aforementioned issues gives valuable information for the DSO in the context of the long-term planning of LV grids. It allows indeed identifying the potential limit violations stipulated in the considered regulation [2]-[3] with regard to the secure network operation and the quality of power supply as well as the critical locations that potentially need to be reinforced.

In this work, the aim is to statistically evaluate the impacts on the modern distribution grid equipment of the changing load profile due to the massive integration of dispersed generation (DG) and the increased load at the LV level. Such a study is vital for the DSO in order to avoid unexpected investments due to a premature aging of the network facilities.

The methodology relies on a probabilistic model based on quarterly energy flows coming from smart meters installed at some customers [4]-[5]. Although these smart meters are expected to be widely deployed in the future, this data 
gathering is still limited to some sparse areas of the distribution grids. Compared to the existing bibliography regarding the development of planning tools in the context of the massive integration of PV installations in the LV grids [6-14], the approach developed by the authors presents as main asset to model the statistical behavior of the customers directly on the basis of smart meter measurements. The accuracy of the developed probabilistic model will therefore be highly improved. It allows indeed avoiding the use of solar irradiations data for modeling the PV panels and weather data combined with synthetic load profiles for the consumption loads. Such an approach does not account for the efficiency of the PV cells and are based on the strong assumption that the load profiles of all LV consumers evolve similarly.

The papers firstly focuses on the principle of the developed process with a particular interest in the utilization of smart meter data. Then, the influence on the network equipment of the integration of PV generation and charging station of EVs at the client level is studied. This work aims thus at determining the power flows in the feeders and at the $\mathrm{MV} / \mathrm{LV}$ substation in order to evaluate their impact on the line congestions and on the transformer aging. Excessive power flows can indeed be destructive for the network facilities and induce unintended expenses for the DSO. The study is carried out on an existing Belgian grid with two feeders and twenty-six residential customers. Finally, conclusions and perspectives are presented.

\section{Exploitation of the smart meter data}

The proposed method relies on energy flows recorded by smart meters installed at some customers. The deployment of these devices was initiated by the apparition of issues such as overvoltages and local congestions caused by the introduction of PV units in residential households. Today, most of the smart meters are thus installed in some selected area characterized by a high penetration rate of DGs (around $25 \%$ ). For those clients with PV units, two smart meters are installed in order to discern contributions of load and generation and to determine useful information for the DSO such as the auto-consumption rate of the prosumer (consumer with its own PV installation) [4]. The installation diagram is presented in Fig. 1. Practically, the energy flows are measured and logged for every time-step of fifteen minutes. Such a time interval will thus be employed between two consecutive simulations.

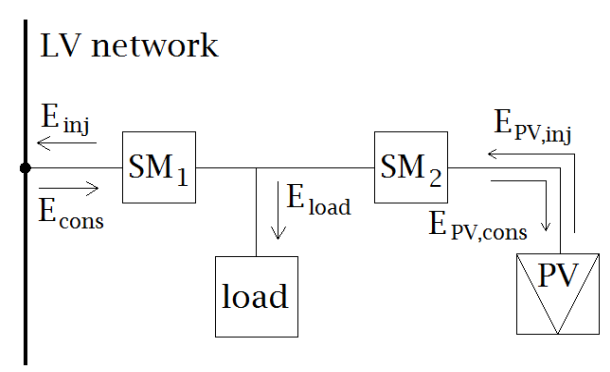

Fig. 1. Typical smart meter installation for a prosumer.

\section{Developed methodology}

The general principle of the method is presented in Fig. 2. Firstly, the data are collected for each customer and classified according each considered "typical day". In this work, twelve "typical days" are defined, one for each month of the year. Naturally, other division requiring more complicated data processing strategy could also be envisaged. Then, based on the energy flows, a cumulative distribution function (CDF) is constructed for each client for the ninety-six quarters of an hour of the twelve "typical days". The prosumers are characterized by two CDFs, one for the consumption and the other one for the energy injected towards the network. As only the power exchanged with the LV grid is of interest for the network operation, the statistical behaviors are built with the data coming exclusively from the SM1.

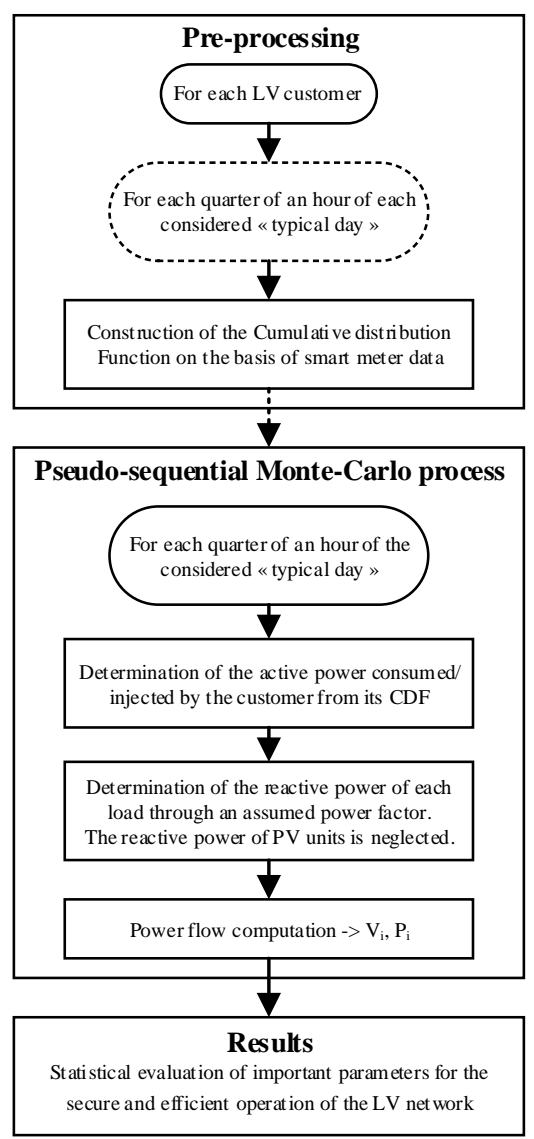

Fig. 2. Flowchart of the general principle of the long-term planning tool.

The probabilistic model used is based on a pseudochronological Monte-Carlo (M-C) algorithm. Indeed, the current lack of large-scale measurements does not allow covering all possible statistical behaviours of LV customers. This method has therefore been privileged compared to other analytical techniques because of its excellent trade-off between simplicity of implementation, accuracy of results and computing time. Concerning the latter, it should be noted that the calculations can be performed in parallel due to the independence of the simulated states.

The procedure relies on a repeated random sampling of the quarter-hourly energy flow of the different LV customers. To that end, a sampling of an uniformly distributed number $u$ on the interval $[0,1]$ is performed and the active power corresponding to that number is inferred from the associated CDF [4]. In order to obtain a good convergence threshold $(<0.1 \%)$ on the results, it is shown that 10,000 
simulations is a good trade-off for keeping a reasonable computational burden [9]. The sampled values are each time introduced in a power flow computation software for radial distribution grids with dispersed generation. This computation yields the voltage profile and the power flow in each line of the simulated LV grid. It should be noted that the reactive power of individual loads is assumed to vary proportionally with the active power (according to a defined power factor) whereas the reactive power of PV units is neglected.

\section{Definition of the studied parameters}

In previous studies, this tool was used for assessing the respect of technical constraints (voltage level, voltage imbalance...) in grids in the presence of a large number of PV installations [4]-[5]. However, it is also of interest for the DSO to compute the power flows in the different feeders as well as in the MV/LV substation as it has a direct impact on the lifetime of the equipment. Furthermore, it is important to already take the probable future massive introduction of EVs into account in the simulations.

In this paper, three scenarios are therefore envisaged. In the first one, the traditional model without PV units and EV charging devices is considered and will constitute the reference scenario. Then, in the second one, the influence of the massive introduction of DGs in LV grids is evaluated. Finally, in the third scenario, the impact of the potential widespread integration of EVs battery charging is examined. It should be noted that no EVs charging devices are practically installed in the studied grid. Nevertheless, these loads are simulated based on typical EV charging profiles and statistical behaviours of other EVs owners.

\section{A. Congestion issues}

The power cables are characterized by their current carrying capacity that defines the maximum current that can flow in the conductors without inducing excessive heating. Indeed, the current flowing in the conductors generates heat that need to be distributed in the surrounding environment. But this diffusion is made difficult by the important thermal resistances of the insulating materials in cables and by the surrounding environment, especially in the case of underground cables. As responsible of the proper operation and the maintenance of the distribution grid, DSOs must make the proper arrangements for avoiding a premature aging or even the melting of the material. The first step is therefore the assessment of the potential risks, task that is undertook in this work.

\section{B. Transformer aging}

The current MV/LV transformers are designed according to the traditional consumption of LV consumers such as residential households and small and medium sized enterprises (SMEs) so that lifetime issues rarely occur. But the current progressive introduction of more important loads by LV consumers such as electrical vehicles (EVs), electric heating (heat pumps) has a significant impact on the temperature of the distribution transformers that can shorten their life expectancy. In this context, the massive integration of photovoltaic (PV) panels could alleviate the influence of such an increase of the load thanks to a local consumption of the generated power. However, the current pricing of day/night meters which financially encourages the night consumption combined with the necessity for customers that some processes like EVs charging have to operate overnight when there is no PV generation could lead to unintended overload situations. This situation is accentuated by the usual evening peak consumption mainly due to cooking processes involving powerful appliances. It is thus essential to implement solutions such as active demand management in order to overcome this problem and, as a result, to prevent the transformer aging. The aging of the transformer is computed based on the hottest spot temperature. Most of the MV/LV substations in the Belgian grid are oil-immersed current transformers. It has been determined experimentally that the aging depends on the time and the temperature and the accelerating factor $F_{A A}$ is then defined by the following empirical formula [17]:

$$
F_{A A}=\exp ^{\left[\frac{15000}{383}-\frac{15000}{\Theta_{H}+273}\right]}
$$

where $\boldsymbol{\Theta}_{\boldsymbol{H}}$ is the hottest spot temperature. The normal life expectancy for such a transformer is the expected lifetime for a constant hot-spot temperature of $110{ }^{\circ} \mathrm{C}$. It corresponds to a lifetime of approximately 20.55 years. The curve representing the acceleration of the aging in function of the temperature is plotted in Fig. 3.

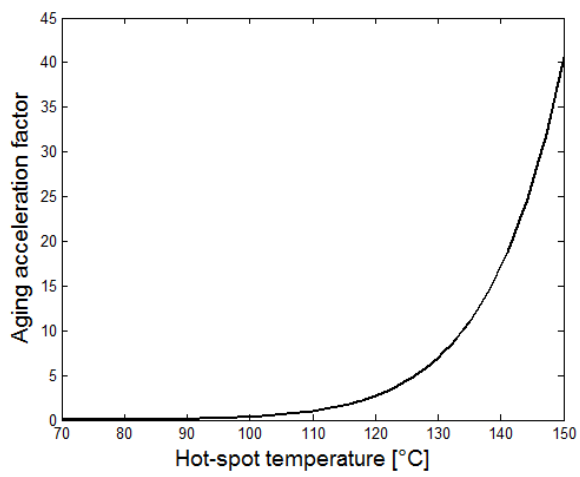

Fig. 3. Relation between the transformer aging acceleration factor and the winding hottest spot temperature.

One can see that the rate at which the insulation deteriorates is very sensitive to a change in temperature. A doubling of the $F_{A A}$ is indeed obtained for a temperature of $116.9{ }^{\circ} \mathrm{C}$. However, if only short periods of high temperature are observed, those can be counterbalanced by long periods of under-loading such that the global lifetime is satisfactory. Moreover, there is a delay between changes in the transformer load and the subsequent impact on the temperature that can smooth the adverse effects of loading peaks. This dynamic has been modeled in [17] with the introduction of two time constants. The first one reflects the top-oil rise over the ambient temperature with the fluctuations of the load and is usually several hours long. The second is in the order of a few minutes and relates to the winding hot-spot rise due to the heating process coming from $R I^{2}$ losses and the heat transfer between the winding and the surrounding oil [18]. These two time constants as well as the current flow in the MV/LV substation computed in the load-flow algorithm are used for determining the transformer hot-spot temperature $\Theta_{H}$. 
The computation consists in the summation of three contributions [17]:

$$
\Theta_{H}=\Theta_{A}+\Delta \Theta_{T O}+\Delta \Theta_{H}
$$

where $\Theta_{A}$ is the ambient temperature, $\Delta \Theta_{T O}$, the top-oil rise over ambient temperature and $\Delta \Theta_{H}$, the winding hottestspot rise over top-oil. It should be emphasized that the values of $\Delta \Theta_{T O}$ and $\Delta \Theta_{H}$ accounts for the variations of the load and of the temperature within the transformer. Furthermore, as time constants are also taken into account, the transition between two consecutive states needs to be physically realistic, which justifies the importance of using a pseudo-sequential algorithm based on real energy flows measurements.

As the time resolution for the aging factor is fifteen minutes for each time-step, the equivalent life can then be calculated with the following equation [17]:

$$
F_{E q A}=\frac{\sum_{n=1}^{N} F_{A A_{n}} \cdot \Delta t_{n}}{\sum_{n=1}^{N} \Delta t_{n}}=\frac{\sum_{n=1}^{N} F_{A A_{n}}}{N}
$$

where $N$ is the number of quarter of an hour simulated. For each of the twelve defined "typical days", this number can be found as follows:

$$
N=96 . N_{d}
$$

where $N_{d}$ is the number of days of the considered month and 96 stands for the number of quarters of an hour in a day. Practically, in this study, the equivalent life $F_{E q A}$ is computed for each of the twelve "typical days". The twelve values are then averaged in proportion to the number of days of each month in order to obtain a value representative of a full year. For each month, the computation is performed in the pseudo-sequential Monte-Carlo algorithm until a satisfying level of convergence $\beta(\beta<1 \%)$ on the value of $F_{E q A}$ is obtained.

\section{Study case and simulation results}

The simulated grid (Fig. 4) is an existing LV network located in Belgium that consists of two feeders with twentysix clients connected to it. The main feeder of the first line is 763 meters long and is composed of nineteen nodes, five of which are prosumers. The total length of the second feeder is 408 meters for seven nodes and two prosumers. The peak rated power of the $5 \mathrm{PV}$ installations vary between $2.65 \mathrm{kVA}$ and $5 \mathrm{kVA}$, namely the maximum power that can be legally installed on a single phase of the Belgian grid.

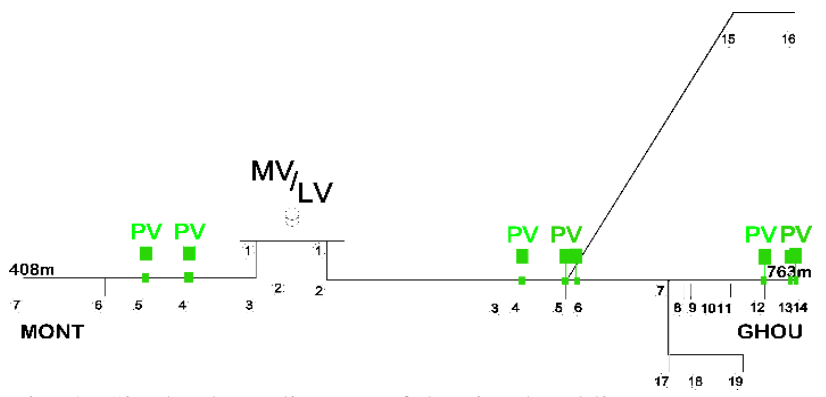

Fig. 4. Single phase diagram of the simulated lines.
Practically, the first scenario considers a traditional LV grid without generation such as PV units. Indeed, the line cables as well as the MV/LV transformer are dimensioned according to this situation. In this way, the rated power of the distribution transformer is $120 \mathrm{kVA}$, which ensured a reasonable security margin even for consumption peaks in traditional distribution grids. In the second scenario, the integration of PV units is envisaged. The studied topology is the one actually observed currently in the considered Belgian LV network. The penetration rate, that is defined as the ratio of the number of households with PV panels with the total number of clients, is about $30 \%$. Finally, in the third scenario, EVs are integrated in the same households that those with PV panels.

Firstly, the probability of congestion is computed for each line section of the grid. The computation is based on the thermal limits of the cables provided by the Belgian DSO responsible of this area. The maximum current that can securely flows within the cables is given in the manufacturer datasheet [19]. For the considered LV network, it corresponds to a maximum apparent power of $64.4 \mathrm{kVA}$. The results for the three scenarios are represented in Fig. 5 for the month of January (maximum load and minimum generation) and Fig.6 for the month of June (maximum generation). These are limited to the first line as no congestion are observed in the second line due to the low number of connections.

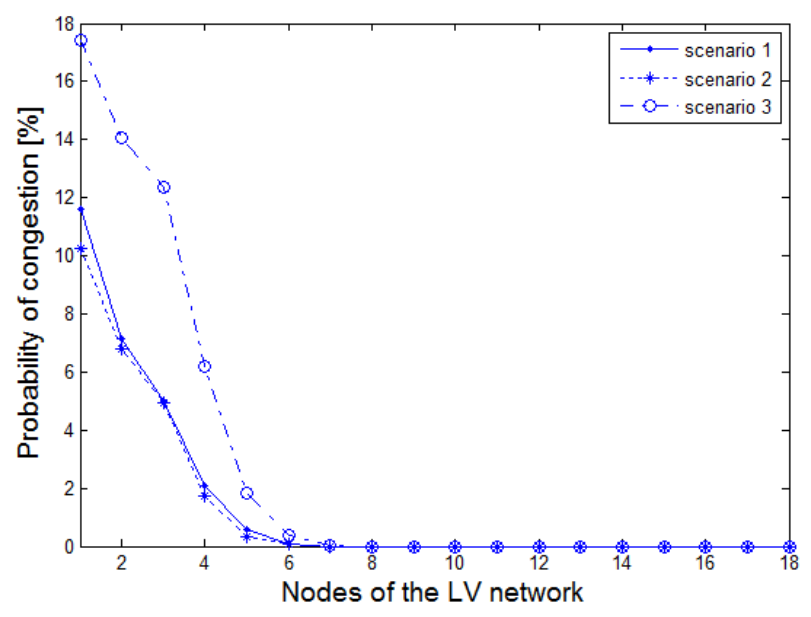

Fig. 5. Probability of congestion for each line section of the first feeder with 19 nodes for the month of January.

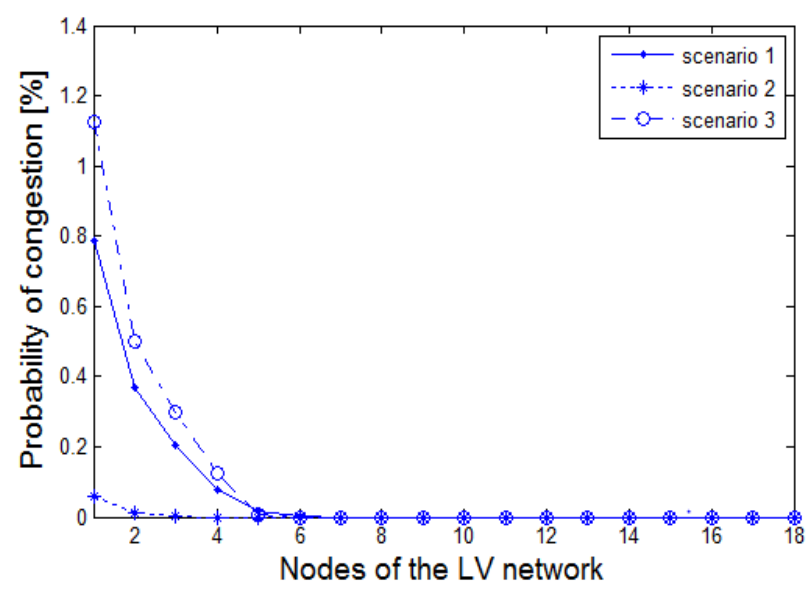

Fig. 6. Probability of congestion for each line section of the first feeder with 19 nodes for the month of June. 
One can see that the number of congestion decreases when generation is included into the grid. This can be explained by the fact that the power produced by these units is consumed locally by the neighbouring loads. Conversely, the introduction of charging devices for electric vehicles in LV grids has an adverse impact on the power flows. Indeed, these are powerful appliances $(230 \mathrm{~V}, 16 \mathrm{~A})$ that significantly increase the current in the different lines.

The yearly loading distribution at the MV/LV substation is now studied. The results are illustrated in Fig. 7.

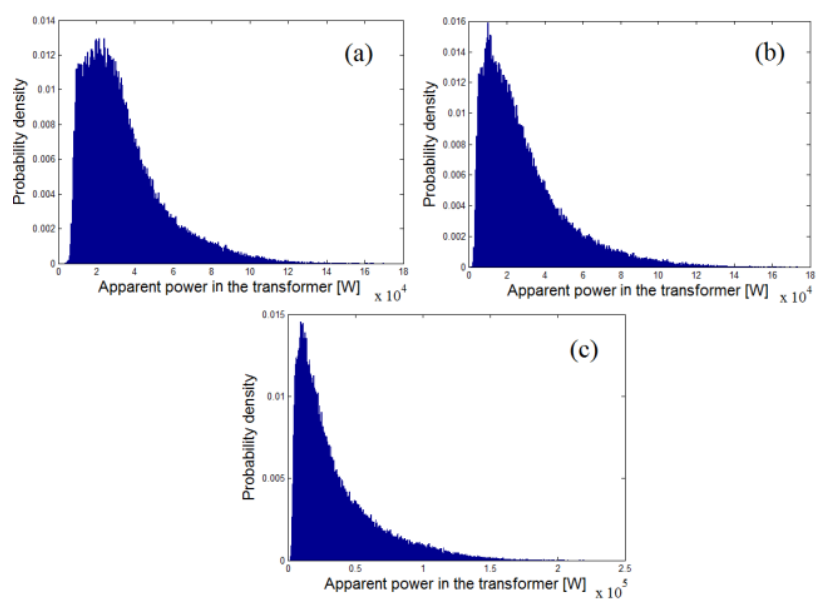

Fig. 7. Yearly loading distribution at the transformer for the traditional LV grid (a), current grids with PV units (b) and future grids with the introduction of EV charging devices (c).

The three distribution functions follow the same pattern but, consistently with the previous observations, the power at the $\mathrm{MV} / \mathrm{LV}$ substation decreases when generation is introduced into the grid and substantially rises with EVs charging appliances.

Finally, the probabilistic study of the impact on the aging of the transformer is outlined in Fig. 8.

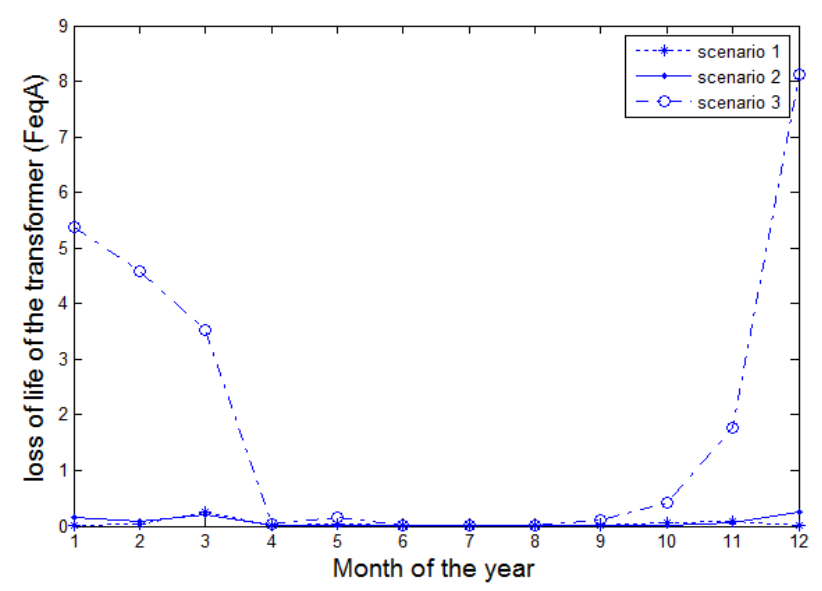

Fig. 8. Monthly averaged loss of life of the MV/LV transformer.

The transformers are currently sized according to the traditional consumption customs in which the main part of the load comes from kitchen appliances. The simulations even highlighted that the transformer of the considered grid is oversized for this situation. Furthermore, the introduction of PV units also had a beneficial impact on the lifetime of the network equipment. However, if the emergence of electric vehicles is notable, the repercussions on the operation of the grid can be damaging. For the studied grid, the global aging of the transformer $\left(F_{E q A}\right)$ averaged for a typical year (critical winter months are counterbalanced by summer months) is around twice the one defined in the IEEE standards [17].

It is therefore of interest for DSO to have probabilistic tools for determining the critical parts of the distribution grid that need to be upgraded. Furthermore, in the case in which the replacement of the equipment is required, the information given by this probabilistic tool can be used for choosing the most appropriate device as regards the rated power according to the current loading situation of the network.

\section{Conclusion and perspectives}

The massive rolling out of smart meter devices in the near future will allow DSOs gathering useful information with regard to the operation of LV grids. This paper was devoted to the development of a probabilistic methodology based on the energy data directly collected by these devices.

This study highlighted the potential issues on the line cables and the MV/LV substation if there is a significant emergence of the electric vehicle in the future. It is therefore essential that DSOs dispose of such probabilistic tools for determining the critical parts of the grid in which upgrades or replacements of the equipment are required.

\section{References}

[1] P. T. Staats, W. M. Grady, A. Arapostathis, R. S. Thallam, "A Procedure for Derating a Substation Transformer in the Presence of Widespread Electric Vehicle Battery Charging", IEEE Trans. on Power Delivery, Vol. 12, No. 4, Oct. 1997.

[2] Voltage characteristics of electricity supplied by public electricity networks, EN 50160, 06.2012.

[3] The Norwegian directive on quality of supply, The Norwegian Water Resources and Energy Directorate, 31 December 2011.

[4] F. Vallée, V. Klonari, T. Lisiecki, O. Durieux, F. Moiny, J. Lobry, "Development of a probabilistic tool using Monte Carlo simulation and smart meters measurements for the long term analysis of low voltage distribution grids with photovoltaic generation", Electrical Power Systems Research,53, pp.468-477, 2013.

[5] V. Klonari, F. Vallée, O. Durieux, Z. De Gréve, J. Lobry, "Probabilistic modeling of short term fluctuations of photovoltaic power injection for the evaluation of overvoltage risk in low voltage grids", in Proc. IEEE International Energy Conference (ENERGYCON), 13-16 May 2014.

[6] S. Conti, S. Raiti, G. Tina, U. Vagliasindi, "Integration of multiple PV units in urban power distribution system”, Solar Energy, 75 (2), pp.87-94, 2003.

[7] S. Conti, S. Raiti, "Probabilistic load flow using Monte Carlo techniques for distribution networks with photovoltaic generators", Solal Energy, 81(12), pp.1473-81, 2007.

[8] F.J. Ruiz-Rodriguez, J.C. Hernandez, F. Jurado, "Technical impact of photovoltaic distributed generation on radial distribution systems: stochastic simulations for a feeder in Spain", Electrical Power Systems Research, 50, pp.25-32, 2013.

[9] F.J. Ruiz-Rodriguez, J.C. Hernandez, F. Jurado, "Probabilistic load flow for photovoltaic distributed generation using the Cornish-Fisher expansion”, Electrical Power Energy Systems, 89, pp.129-38, 2012.

[10] F.J. Ruiz-Rodriguez, J.C. Hernandez, F. Jurado, "Probabilistic load flow for radial distribution network with photovoltaic generators", IET Renewable Power Generation, 6(2), pp. 110-121, 2012.

[11] P. Zhang, W. Li, S. Li, Y. Wang, W. Xiao, "Reliability assessment of photovoltaic power systems: review of current status and future perspectives", Applied Energy, 104, pp.822-33, 2013. 
[12] R. Billinton, B. Bagen, "Generating capacity adequacy evaluation of small standalone power systems containing solar energy", Reliability Engineering \& System Safety, 91(4), pp.438-43, 2006.

[13] R. Billinton, R. Karki, "Reliability/cost implications of utilizing photovoltaics in small isolated power systems", Reliability Engineering \& System Safety, 79(1), pp.11-16, 2003.

[14] R.M. Moharil, P.S. Kulkarni, "Reliability analysis of solar photovoltaic system using hourly mean solar radiation data", Solar Energy, 84(4), pp.691-702, 2010.

[15] B. Meersman, B. Renders, L. Degroote, T. Vandoorn, L. Vandevelde,

"Three-phase inverter-connected DG-units and voltage unbalance", Electric Power Systems Research 2011:81:899-906.

[16] M. Karahan, O. Kalenderli, "Coupled Electrical and Thermal Analysis of Power Cables Using Finite Element Method", Heat transfer Engineering Applications, 2011.

[17] IEEE Guide for Loading Mineral Oil-Immersed Transformers, IEEE Standard C57.91-1995.

[18] J. Perez, "Fundamental principles of transformer thermal loading and protection", 63rd Annual Conference for Protective Relay Engineers, 2010 .

[19] Nexans, "Câbles de réseau basse tension et câbles moyenne tension », 2006.

\section{Acknowledgement}

This work is being supported in terms of smart metering data supply by ORES, the DSO who is in charge of managing the electricity and natural gas distribution grids in 196 communes in Wallonia, in Belgium. 